\title{
Características da lactação de vacas Hereford criadas em um sistema de produção extensivo na região da campanha do Rio Grande do Sul ${ }^{1}$
}

\author{
Marcelo Alves Pimentel ${ }^{2}$, José Carlos Ferrugem Moraes ${ }^{3}$, Carlos Miguel Jaume ${ }^{3}$, Jaqueline \\ Schneider Lemes ${ }^{4}$, Cássio Cassal Brauner ${ }^{4}$
}

\footnotetext{
${ }^{1}$ Parte da tese de Doutorado do primeiro autor apresentada ao Programa de Pós-Graduação em Zootecnia, Faculdade de Agronomia Eliseu Maciel, Universidade Federal de Pelotas, como parte das exigências para obtenção do título de Doutor em Ciências.

2 FAEM - UFPEL, Caixa Postal 354, Pelotas - RS, CEP: 96010-900.

${ }^{3}$ Embrapa Pecuária Sul.

${ }^{4}$ Graduação em Veterinária - UFPEL.
}

RESUMO - A produção de leite de vacas Hereford primíparas e multíparas manejadas sobre campo nativo foi estudada utilizando-se 144 lactações em um período de quatro anos (1995, 1998, 2000, 2001). A estimativa da produção de leite foi realizada em intervalos de 21 dias pela técnica da pesagem antes e após a mamada, do nascimento à desmama (189 dias). Foram considerados os fatores fixos ano, ordem de parto, prenhez, sexo do bezerro e raça do touro. As vacas multíparas produziram mais leite $(8,27 \pm 0,26)$ que as primíparas $\left(5,81 \pm 0,25 \mathrm{~kg} \mathrm{dia}^{-1}\right)$ no pico de lactação (42 dias). A produção total de leite em multíparas também foi superior $(158,34 \pm 25,51)$ à das primíparas $(915,44 \pm 24,47 \mathrm{~kg})$. A produção de leite não foi influenciada pelos demais fatores. A maior produção de leite durante o pico de lactação consiste na principal diferença entre as curvas de lactação de vacas multíparas e primíparas, que, no entanto, apresentam amplitude de produção de leite similar nas condições estudadas. O leite fornece a energia suficiente para atender às exigências de mantença e ganho dos bezerros durante os dois primeiros meses da lactação e, apenas para mantença, até o quarto mês de lactação.

Palavras-chave: bovinos de corte, produção de leite

\section{Lactation performance of Hereford cows raised in a range system in the state of Rio Grande do Sul}

\begin{abstract}
Milk yield of primiparous and multiparous Hereford cows grazing native pastures was studied using 144 lactations from four different years $(1995,1998,2000,2001)$. Estimates of milk yield were obtained every 21-day interval from birth to weaning (189 d) by the weigh-suckle-weigh technique. Effects of year, calving order, pregnancy, calf sex, and sire breed were studied. Multiparous cows $(8.27 \pm 0.26 \mathrm{~kg} / \mathrm{d})$ yielded more milk than primiparous cows $(5.81 \pm 0.25 \mathrm{~kg} / \mathrm{d})$ at peak of lactation $(42 \mathrm{~d})$. Total milk yield also was higher for multiparous $(1,158.34 \pm 25.51 \mathrm{~kg} / \mathrm{d})$ compared to primiparous $(915.44 \pm 24.47 \mathrm{~kg} / \mathrm{d})$. Therefore, the main difference between multiparous and primiparous lactation curves was production at peak although similar lactation persistence was found. All remaining effects did not affect milk yield in the present trial. Milk met calf requirements for maintenance and growth in the first 2 months of lactation and only maintenance requirements until the fourth month.
\end{abstract}

Key Words: beef cattle, milk yield

\section{Introdução}

Estudos sobre produção de leite em vacas de corte datam de muito tempo, caracterizando a produção e composição (Cole \& Johansson, 1933) e, até mesmo, relacionando-a a características produtivas como qualidade da carcaça de novilhos (Cook et al., 1942). Atualmente, têm-se realizado estudos com diferentes focos, relacionando produção de leite em vacas de corte com crescimento e comportamento do bezerro (Neville, 1962; Melton et al., 1967; Rutledge et al., 1971; Boggs et al., 1980; Bartle et al., 1984; Alencar et al.,
1985; Alencar, 1987a; Alencar et al., 1996; Day et al., 1987; Franzo et al., 1997; Buchanan \& Stutts, 2001), com eficiência reprodutiva pós-parto (Bartle et al., 1984; Alencar, 1987b; Beal et al., 1990; Buchanan et al., 2000; Pimentel et al., 2002), com a idade das vacas (Rutledge et al., 1971; Neville et al., 1974; Robison et al., 1978; Boggs, 1980), com medidas corporais (Williams et al., 1979b) e com os efeitos de genótipo, nutrição, época de parição e eficiência de sistemas de produção (Melton et al., 1967; McMorris \& Wilton, 1986; Green et al., 1991; Jenkins \& Ferrel, 1992; Miller et al., 1999; Silveira et al., 1999; Jenkins et al., 2000; Pimentel et al.,

Correspondências devem ser enviadas para: map@ufpel.tche.br 
2001; Mendonça et al., 2001). Contudo, permanece ainda o interesse em verificar a quantidade de leite que pode ser produzida por uma vaca de corte sob condições extensivas, pois o conhecimento da produção, das características da curva de lactação e dos fatores que interferem no processo pode contribuir para determinação de práticas de manejo e recomendações técnicas relacionadas ao desempenho produtivo de vacas e bezerros (Leal \& Freitas 1982, Alencar, 1987b, 1996).

A produção de leite em vacas de corte pode ser mensurada no ato da ordenha, manual ou mecânica, após injeção intravenosa de 20 a 40 UI de ocitocina (Totusek \& Dudley, 1965; Wistrand \& Riggs, 1966; Jeferry et al., 1971; McMorris \& Wilton, 1986; Lalman et al., 2000; Johnson et al., 2003) ou pelo método da pesagem do bezerro antes e após a mamada (Dillard et al., 1978, Williams et al., 1979a). Segundo Beal et al. (1990), apesar de possuir repetibilidade $(0,35)$ menor que o método que utiliza a ordenha mecânica $(0,97)$, ambos têm correlação alta e similar $(>0,75)$ com o ganho de peso dos bezerros antes da desmama, sendo o método da pesagem-mamada-pesagem o mais indicado para investigações com grande número de vacas.

A lactação é um estado fisiologicamente dominante e homeorético (Bauman, 2000) no qual ocorrem adaptações no metabolismo, em alguns órgãos e tecidos e no comportamento da vaca para a síntese do leite. Em vacas de corte, a produção de leite é, em parte, limitada pela capacidade de alimentação do bezerro e a produção máxima ocorre em torno do segundo mês de lactação (Drewry et al., 1959). Estudos sobre comportamento indicam que a freqüência de amamentação tende a diminuir com o avançar da idade do bezerro e que a duração da amamentação tende a aumentar e o tempo total a diminuir no transcurso da lactação. Bezerros mais novos mamam mais vezes e durante menos tempo e, em produções de leite mais baixas, a tendência é aumentar o tempo de mamada, principalmente até o pico da lactação. A variação da freqüência pode ser grande ( 2 a $\left.23 \mathrm{dia}^{-1}\right)$, com médias de 8,6 mamadas no pico (52 dias) e de 4,5 no final (167 dias) da lactação (Day et al., 1987). Portanto, não só a limitação do bezerro determina a forma da curva de lactação. Além disso, as características de produção de leite da vaca interferem no comportamento do bezerro, de modo que a disponibilidade de leite também pode modificar o hábito alimentar, aumentando ou diminuindo a compensação do aporte de nutrientes pelo consumo de forragem (Boggs et al., 1980). Entretanto, os níveis de produção de leite em vacas de corte e as diferentes curvas de lactação parecem ser limitados, podendo ser afetados por efeitos ambientais e genéticos, como período da lactação (Green et al., 1991), idade da vaca, tipo de alimentação (Neville, 1962; Jenkins et al., 2000), raça ou grupamento genético (Jenkins \& Ferrell, 1992). Quanto ao período da lactação, Drewry et al. (1959) apontam a quarta semana como a de maior produção. Todavia, Franzo (1997), estudando diferentes genótipos de vacas, verificou que o pico de lactação ocorria na mesma época do ano, porém, com diferentes períodos de tempo (63 e 84 dias). Green et al. (1991) observaram produções máximas de 8,26 a $10,38 \mathrm{~kg}$ no período de 53 a 68 dias em quatro grupos raciais, enquanto Jenkins et al. (2000) verificaram picos de lactação na $6^{\mathrm{a}}$ semana, com produções de 7 a $9 \mathrm{~kg}$, em seis raças. Jenkins \& Ferrell (1992), no entanto, constataram que acréscimos no nível energético da dieta correspondem a aumentos na duração e média de produção de leite no pico da lactação.

As vacas de corte apresentam maiores produções de leite no início da lactação, com um declínio até o desmame, tornando a persistência da produção uma variável importante (Neville, 1962, Alencar et al., 1995), pois expressa o quanto reduz o leite produzido a partir do pico da lactação. Green et al. (1991) compararam a persistência da lactação em diferentes genótipos utilizando a relação entre a produção total e o pico da lactação e observaram que, quanto maiores os valores obtidos, maior a persistência. A redução média diária, medida pela diferença entre a última produção e o pico $(\mathrm{g})$, ponderada pelo intervalo de tempo (dias), foi utilizada por Jenkins et al. (2000), que verificaram valores de $-41,0$ e $-52,1 \mathrm{~g} / \mathrm{dia}$ em seis raças. Consta na Tabela 1 a produção de leite de vacas de corte reportada por diferentes autores.

O efeito da produção de leite sobre o bezerro, contrariamente à vaca, apresenta relação favorável e está diretamente relacionado ao seu desempenho na fase de cria. O peso do bezerro à desmama é determinado, em grande parte, pelo consumo de leite (60 a 66\% da variação) (Drewy et al., 1959; Neville, 1962; Rutledge et al., 1971).

Quanto à eficiência de utilização do leite, Alencar(1987a) verificou que filhos de vacas boas produtoras apresentam menores ganhos e que bezerros que tomam mais leite são mais pesados à desmama. Constatou ainda que, quanto maior a produção de leite da vaca, maior a quantidade de leite necessária para ganho de 1,0 kg de peso, que bezerros mais pesados ao nascer são menos eficientes na utilização do leite consumido e que a eficiência na utilização do leite é maior em bezerros filhos de vacas que produzem menos leite, principalmente após o primeiro mês de vida. Da mesma forma, Neville (1962) observou que bezerros de vacas em melhores condições nutricionais necessitam de maior quantidade de leite para produzir uma unidade de ganho de peso dos 4 aos 8 meses de idade. 
Tabela 1 - Médias de produção diária de leite $(\mathrm{kg})$ durante os meses de lactação, utilizando-se o método pesagem-mamada-pesagem Table 1 - Means of daily milk yield $(\mathrm{kg})$ during different months of lactation obtained with weight-suckle-weight method

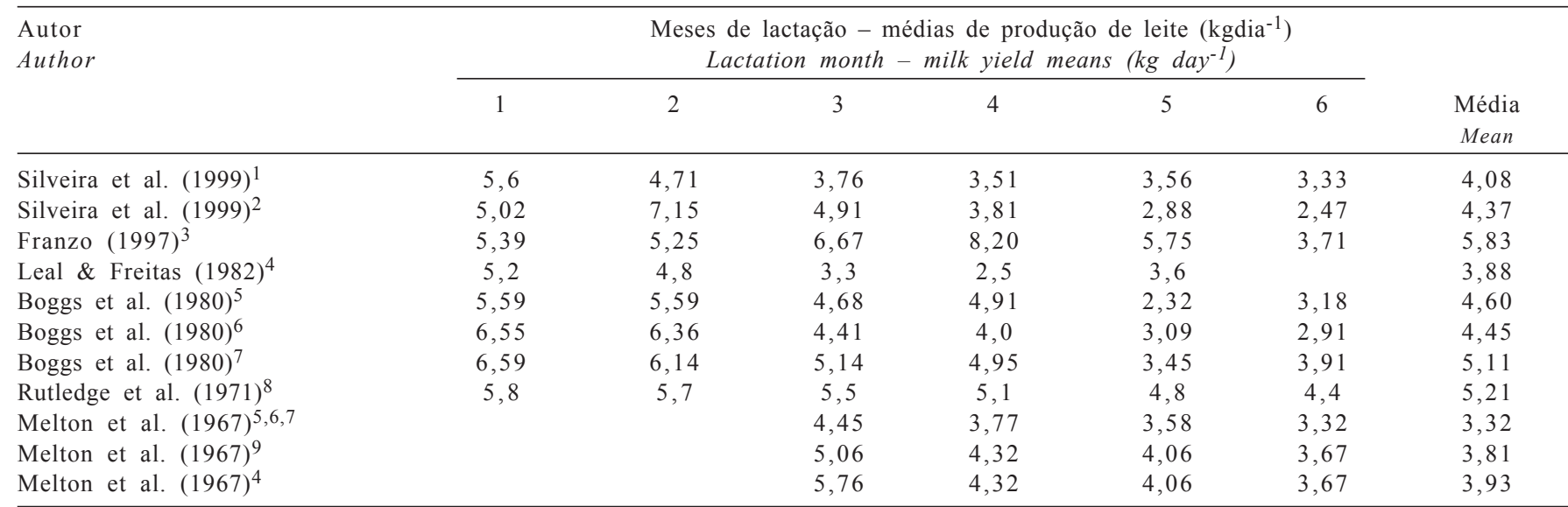

${ }^{1}$ Hereford parição primavera, ${ }^{2}$ Hereford parição outono ${ }^{3}$ Cruza 3 anos, ${ }^{4}$ Charolês, ${ }^{5}$ Hereford 3 anos, ${ }^{6}$ Hereford 4 anos, ${ }^{7}$ Hereford $5-8$ anos, ${ }^{8}$ Hereford 2-15 anos, 9 Angus 2 a 10 anos

${ }^{1}$ Hereford spring calving, ${ }^{2}$ Hereford fall calving, ${ }^{3}$ Crossbred 3 years old, ${ }^{4}$ Charolais, ${ }^{5}$ Hereford 3 years old, ${ }^{6}$ Hereford 4 years old, ${ }^{7}$ Hereford $5-8$ years old, ${ }^{8}$ Herfeford $2-15$ years old, ${ }^{9}$ Angus 2-10 years old.

Robison et al. (1978) verificaram que até o quarto mês de lactação o leite supre, com sobra para ganho de peso, as necessidades de energia líquida dos bezerros, porém, apresenta déficit no quinto e sexto meses. Estudando a complementação entre leite e forragem, Boggs et al. (1980) afirmaram que o consumo de forragem aumenta de 0,44 para $3,52 \mathrm{~kg} /$ bezerro/ dia, representando 0,$62 ; 1,46 ; 1,51 ; 1,75$ e $2,20 \%$ do PV para bezerros com 2, 3, 4, 5 e 6 meses de vida, respectivamente. A quantidade de leite requerida por unidade de ganho de peso diminui com a idade do bezerro e cada quilo a mais de leite consumido por dia representou $7,2 \mathrm{~kg}$ no peso à desmama $\mathrm{e}$ $0,34 \mathrm{~kg}$ no GMD. De modo geral, os autores demonstraram que o consumo de leite tem efeito negativo sobre o consumo de forragem dos 2 aos 6 meses de idade, que bezerros com dois meses consomem $0,3 \mathrm{~kg} \mathrm{dia}^{-1}$ amenos de forragema cadakg deleite consumido e que aos seis meses essa relação é de $0,7 \mathrm{~kg} \mathrm{dia}^{-1}$.

Considerando a problemática das produções de leite e de carne, este estudo foi realizado com o objetivo de caracterizar a lactação de vacas de corte da raça Hereford, primíparas e multíparas, criadas sob condições extensivas de produção na região da campanha do Rio Grande do Sul.

\section{Material e Métodos}

Foram avaliadas 144 lactações em um rebanho de bovinos da raça Hereford. As observações foram realizadas durante quatro anos $(1995,1998,2000$ e 2001) em parições ocorridas na primavera (setembro, outubro e novembro). Nos dois primeiros anos, foram utilizadas novilhas primíparas com média de 36 meses de idade ao parto, e, nos anos seguintes, vacas adultas de 5 a 7 anos de idade, com exceção do ano de 2001, no qual foram utilizadas quatro primíparas, totalizando 23, 48, 42 e 31 animais, respectivamente, para os quatro anos. No primeiro ano, os bezerros eram filhos de touro Hereford; no segundo, 16 de Red Angus e 32 de Nelore; no terceiro, todos de touros Nelore; e, no quarto ano, oito de touros Nelore e 23 de Hereford. Em todos os experimentos, os animais foram mantidos em campo natural, sob lotação de $0,7 \mathrm{UA} / \mathrm{ha}$ (UA $=$ unidade animal $=450 \mathrm{~kg}$ de peso vivo). O trabalho foi conduzido em uma fazenda particular situada no município Aceguá, na região fisiográfica da campanha, no Rio Grande do Sul, localizado entre os paralelos $30^{\circ} 30^{\prime}$ e $31^{\circ} 56^{\prime}$ sul e os meridianos de $55^{\circ}$ $30^{\prime}$ e $54^{\circ} 30^{\prime}$ a oeste de Greenwich.

A região apresenta clima mesotérmico, tipo subtropical da classe Cfa 1, de acordo com a classificação de Köeppen, com as chuvas distribuídas uniformemente durante o ano. A precipitação média anual é de $1.350 \mathrm{~mm}$, variando entre 1.080 e $1.620 \mathrm{~mm}$ (Macedo, 1984), e a temperatura média anual é de $17,6^{\circ} \mathrm{C}$, podendo ocorrer temperaturas extremas de -4 a $41^{\circ} \mathrm{C}$. Geadas podem ocorrer entre os meses de abril e novembro, com maior incidência de junho a agosto. A umidade relativa do ar oscila entre 75 e $85 \%$. O relevo da região de Aceguá é suavemente ondulado, com topografia plana em determinadas áreas. A altitude varia de 80 a $180 \mathrm{~m}$ acima do nível do mar. A fertilidade natural do solo é moderada pelos baixos teores de fósforo e a cobertura natural do solo é superior a $80 \%$, sendo formada por vegetação nativa, com variações qualitativas e quantitativas nos diferentes períodos do ano. A vegetação predominante é a de gramíneas de ciclo estival, formada principalmente pela grama-forquilha (Paspalum notatum) e grama-tapete (Axonopus affinis). As gramíneas de ciclo hibernal de maior ocorrência são as flexilhas (Stipa spp) e o azevém (Lolium multiflorum spp). A população de leguminosas de ciclo hibernal é, na maior parte, composta por trevo (Tripholium spp). 
A produção de leite foi estimada pelo método da pesagem do bezerro antes e após a mamada (Bartle et al., 1984; Beal et al. 1990). Os animais eram separados das vacas no dia anterior ao da medida $(6 \mathrm{~h}) \mathrm{e}$, ao final da tarde ( $18 \mathrm{~h})$, eram colocados para mamar, visando ao esgotamento do úbere. Posteriormente, eram separados por 12 horas. Na manhã seguinte $(6 \mathrm{~h})$, eram pesados em jejum e colocados para mamar durante 20 a 30 minutos e, em seguida, eram pesados, de modo que a diferença entre os pesos foi considerada a produção de leite de 12 horas, que, multiplicada por dois, foi utilizada como a estimativa para 24 horas. As medidas de produção de leite foram realizadas em intervalos de 21 dias, com exceção do experimento do ano 2000, no qual foram realizadas seis medidas de produção - as três primeiras em intervalos de 21 e as demais, de 42 dias. Na primeira avaliação, a idade dos bezerros foi ajustada para 21 dias, utilizando-se as observações relativas a um período de amamentação de 189 dias para os quatro anos. Para as pesagens, foi utilizada balança eletrônica, com capacidade máxima de $1.500 \mathrm{~kg}$ e precisão de $100 \mathrm{~g}$. Vacas e bezerros tiveram seu peso vivo acompanhado do parto ao desmame, com pesagens e avaliação da condição corporal (CC) das vacas (apenas nas vacas adultas) na ocasião da medida de produção de leite.

A produção de leite nos períodos foi estimada pela fórmula proposta por Alencar et al. (1996): $\mathrm{PL}_{\mathrm{n}}=\left[\left(\mathrm{L}_{\mathrm{n}}+\mathrm{L}_{\mathrm{n}-1}\right) / 2\right] \times 21$, em que $\mathrm{PL}_{\mathrm{n}}$ é a produção de leite do período de 21 dias; $\mathrm{L}_{\mathrm{n}}$, a produção de leite estimada do dia, ou seja, a produção observada no dia da pesagem multiplicada por dois; e $\mathrm{n}=21 ; 42 ; \ldots$, 189. A produção de leite total (PLT) de cada vaca foi estimada somando-se todas as $\mathrm{PL}_{\mathrm{n}}\left(\mathrm{PLT}=\sum \mathrm{PL}_{\mathrm{n}}\right)$. A persistência da lactação (PER) foi calculada como a relação entre a diferença da última produção diária $\left(\mathrm{PL}_{9}\right)$ e da mais alta produção verificada(PL2) pelo período, em dias, entre elas: PL9-PL2/147 dias. Esta variável é expressa em $\mathrm{g} /$ dia e significa o quanto se reduz a produção média de leite por dia a partir do pico de lactação (Jenkins et al., 2000).

Nos dois primeiros anos, as vacas primíparas foram acasaladas nos meses de dezembro, janeiro e fevereiro, em monta natural, com relação touro:vaca de 1:25. Nos experimentos seguintes, as vacas adultas foram submetidas à sincronização de cio entre 30 e 100 dias após o parto. Utilizou-se o método descrito por Souza \& Moraes (1998), empregando pessários (esponjas intravaginais impregnadas com $250 \mathrm{mg}$ de acetato de medroxiprogesterona) por sete dias e aplicação de $1 \mathrm{mg}$ de benzoato de estradiol no momento da colocação. Após a retirada dos pessários, separavam-se os bezerros das vacas por quatro dias e executava a inseminação artificial (IA) pelo método convencional, com observação de cio. Seqüencialmente, as vacas foram acasaladas em monta natural por 50 dias. Todas as vacas foram submetidas a diagnóstico de gestação 60 dias após o término do período de acasalamento e as gestantes foram controladas até o parto para se verificar a data da concepção.

Para análise dos resultados, foram considerados fatores fixos ano (A), ordem do parto (OP), resultado do diagnóstico de gestação ou prenhez (P), sexo do bezerro (ST) e raça do touro (RT). Como variáveis-resposta, foram considerados a produção total de leite (PLT), a produção de leite nas medidas diárias (PL), a persistência da lactação (PER), o peso do bezerro ao nascer (PTN), nas pesagens (PT) e ao desmame e o peso corrigido para 189 dias (PTD). Os dados foram submetidos à ANOVA - GLM, no programa NCSS 6.0 (1996), considerando-se o seguinte modelo, descrito conforme a disponibilidade de informações nos distintos anos:

$$
\mathrm{Y}_{\mathrm{ijklm}}=\mu+\mathrm{A}_{\mathrm{i}}+\mathrm{OP}_{\mathrm{j}}+\mathrm{P}_{\mathrm{k}}+\mathrm{ST}_{1}+\mathrm{RT}_{\mathrm{m}}+\mathrm{e}_{\mathrm{ijklm}}
$$

em que $Y_{\mathrm{ijklm}}=$ uma observação de PLT, PL, PER, PTN e PTD, $\mu=$ média geral, $A_{i}=$ efeito do ano $(i=1995,1998,2000,2001)$, $\mathrm{OP}_{\mathrm{j}}=$ efeito da ordem do parto ( $\mathrm{j}=$ primípara, multípara), $\mathrm{P}_{\mathrm{k}}$ $=$ efeito da prenhez $(\mathrm{k}=$ prenhe, vazia $), \mathrm{ST}_{1}=$ efeito do sexo do bezerro ( $\mathrm{l}=$ macho, fêmea), $\mathrm{RT}_{\mathrm{m}}=$ efeito da raça do touro ( $m=$ Hereford, Nelore, Red Angus), $\mathrm{e}_{\mathrm{ijklm}}=$ erro experimental.

\section{Resultados e Discussão}

As médias ajustadas das estimativas de produção de leite nas nove medidas de produção diária (PL) e a média de produção diária da lactação (PLx) são descritas na Tabela 2. As produções foram mais elevadas na segunda e terceira PL, com média geral de 7,04 e 5,91 kg, respectivamente, considerando-se todos os efeitos principais do modelo matemático. Os efeitos de ano e ordem de parto foram significativos $(\mathrm{P}<0,05)$ nessas fases, mas apresentaram-se confundidos, pois, nos primeiros dois anos, as vacas eram primíparas e, nos anos seguintes, multíparas. Considerando apenas o efeito de ordem de parto, as vacas adultas apresentaram médias de produção de leite mais elevadas, corroborando os resultados de outros estudos, nos quais também também foram reportados efeitos da idade da vaca sobre a produção de leite (Drewry et al., 1959; Dawson et al., 1960; Rutledge et al., 1971; Neville et al., 1974; Robison et al., 1978; Boggs et al., 1980; Johnson et al., 2003). As vacas multíparas produziram, aos 42 e 63 dias, respectivamente, $2,46 \mathrm{e} 2,11 \mathrm{~kg}$ a mais $(\mathrm{P}<0,05)$ que as primíparas. Nos demais períodos, a ordem de parto não teve efeito significativo $(\mathrm{P}>0,05)$. Estes resultados indicam que a maior diferença nas características da lactação de vacas primíparas e multíparas ocorre no pico da lactação, entre 42 e 63 dias, ressaltando-se que vacas adultas produzem mais leite, 
Tabela 2 - Médias ajustadas de produção de leite diária e média geral (PLx) nos fatores ano, ordem do parto (VM = multípara, $\mathrm{VP}=$ primípara $)$, prenhez $(\mathrm{P}=$ prenhe, $\mathrm{V}=$ vazia $)$, sexo do bezerro $(\mathrm{M}=$ macho, $\mathrm{F}=$ fêmea $)$ e raça do touro $(\mathrm{N}=\mathrm{Nelore}$, $\mathrm{H}=$ Hereford, RA = Red Angus)

Table 2 - Daily milk yield means and overall mean $(P L x)$ adjusted for, year, calving order $(M=$ multiparous, $P=$ primiparous $)$, pregnancy $(P=$ pregnant, $V=$ non pregnant $)$, calf sex $(M=$ male, $F=$ female) and sire breed $(N=$ Nellore, $H=$ Hereford, $R A=$ Red Angus)

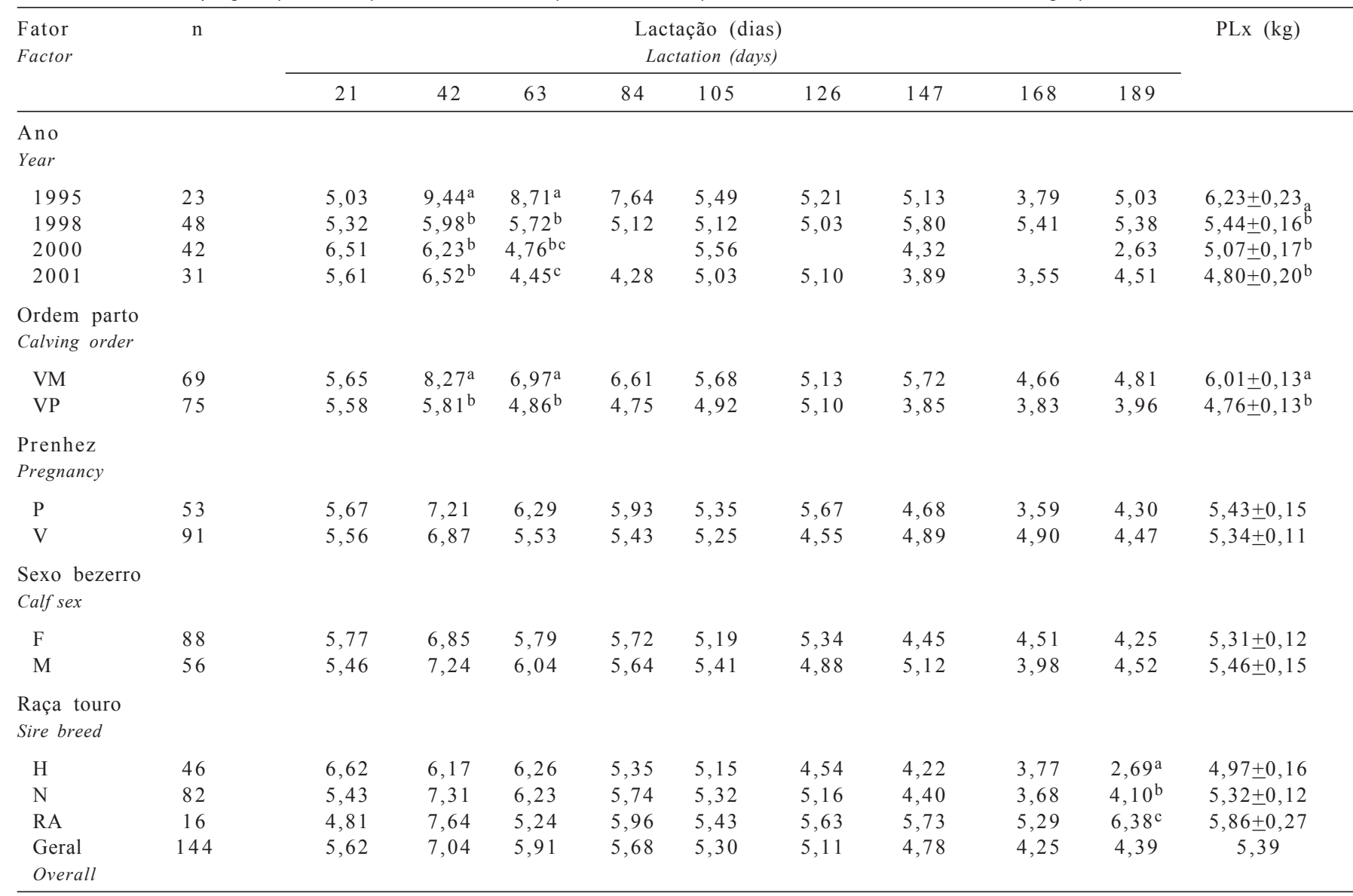

Letras distintas na coluna, dentro de fatores, indicam médias diferentes $(P<0,05)$.

Means followed by different letters in the same column differ $(P<0.05)$.

como verificado por Neville et al. (1974) e Boggs et al. (1980). As linhas de tendência, relativas às médias ajustadas de produção diária de leite (com suas respectivas equações), representando as curvas de lactação de vacas primíparas e multíparas, encontram-se na Figura 1. O pico de lactação ocorreu em torno da sexta semana, coincidindo com o verificado por Jenkins et al. (2000) em seis raças bovinas. Porém, intervalos pouco maiores foram observados por Green et al. (1991), em vacas Hereford x Angus e cruzas com Brahman, Suíço e Pinzgauer (60,3; 68,0; 67,0 e 53,5 dias, respectivamente).

Trabalhando em condições semelhantes às deste estudo, Franzo (1997) somente verificou o pico de lactação aos 84 dias, em novilhas primíparas cruza Hereford x Nelore $\mathrm{x}$ Charolês, com média de 8,2 kg, em lactação de 189 dias (Tabela 1), provavelmente em razão do efeito de época de parição, pois outro grupo de novilhas paridas mais tarde apresentou o pico no mesmo período. Os valores de produção máxima diária verificados no primeiro mês de lactação foram maiores que os apresentados pelos autores citados na Tabela 1, mas as curvas de lactação são semelhantes e se enquadram em uma amplitude característica de vacas de corte, apesar da diferença no material genético e nos sistemas de criação (Melton, 1967; Rutledge et al., 1971; Boggs, 1980; Leal \& Freitas, 1982; Silveira et al., 1999).

Valores relativos ao pico de lactação, à persistência (PER) e à produção total de leite (PLT) encontram-se na Tabela 3. A PLT de vacas adultas foi de $242,89 \mathrm{~kg}$ a mais que nas primíparas $(\mathrm{P}<0,05)$. A ordem de parto não teve efeito significativo $(\mathrm{P}>0,05)$ na persistência da lactação, apesar de o valor verificado para a queda diária de produção das multíparas ter sido quase o dobro do das primíparas.

O efeito nutricional sobre a produção, o tempo do pico de lactação e a produção total foi estudado em nove diferentes raças por Jenkins \& Ferrell (1992) e todas as variáveis aumentaram de forma proporcional ao consumo de energia. O pico de lactação foi entre 8,8 e 11,1 semanas, para Hereford/ Limousin e Red Poll, respectivamente, e as produções foram 


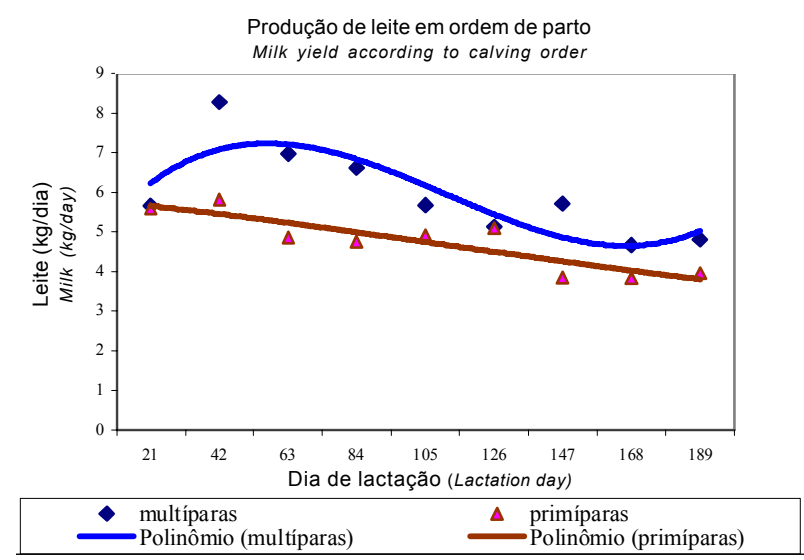

Figura 1 - Curvas de lactação, representadas pelas linhas de tendência das médias diárias ajustadas de produção de leite de vacas multíparas e primíparas.

Figure 1 - Lactation curves represented by tendency lines related to adjusted daily means of milk yield of multiparous and primiparous cows.

entre 8,5 e 11,9 kg para Hereford e Suíço. A produção da raça Hereford foi bem próxima às verificadas neste trabalho, que foi realizado de forma extensiva. Estudando quatro diferentes genótipos, Green et al. (1991) consideraram persistência a relação entre a produção total de leite e a do pico, classificando como de maior persistência raças com picos de lactação mais baixos, quando semelhantes às produções totais. A maior PER nas vacas primíparas, neste trabalho, deve-se fundamentalmente ao seu baixo pico de lactação. Os valores verificados por Jenkins et al. (2000), em lactação de 168 dias, para persistência, são entre - 41,0 e -52,1 g/dia e, portanto, foram menores que os deste estudo. O pico de lactação (42 dias), no entanto, ocorreu na mesma época e apresentou valores semelhantes, entre 7 e $9 \mathrm{~kg}$.

$\mathrm{O}$ efeito da gestação sobre a produção de leite total e durante os nove períodos de avaliação (Tabela 2) não foi significativo ( $\mathrm{P}>0,05)$. Alencar et al. (1993), estudando o intervalo parto-primeiro cio e o intervalo de partos, notaram tendências de efeito negativo da produção de leite sobre a eficiência reprodutiva pós-parto. A influência negativa da produção de leite também é verificada sobre o intervalo parto-primeiro serviço, sugerindo que se determinem experimentalmente os níveis ideais de produção de leite para as raças de bovinos de corte, visando atender ao desenvolvimento do bezerro e à boa eficiência reprodutiva da vaca (Alencar, 1987b). Neste estudo, não foram evidenciadas diferenças na produção de leite entre vacas eficientes e não eficientes, uma vez que o período de acasalamento foi o mesmo para todas. É provável que outros fatores tenham efeito sobre a fertilidade pós-parto com maior magnitude que a produção de leite, pois parece que a limitação característica das raças de corte não favorece uma interferência dessa ordem, ou seja, muitas vezes o efeito da produção de leite é confundido com deficiências na alimentação. A lactação fisiologicamente é prioritária à reprodução (Short et al., 1990). Portanto, sempre, e principalmente no início, a partição de nutrientes deve ser no sentido da produção de leite. A limitação das vacas de corte em produzir leite pode amenizar esse quadro, mas, mesmo dentro dessa limitação, o aumento do consumo de energia pode afetar a produção inicial de leite, que, segundo Lalman et al.(2000), estaria associada a maior intervalo pós-parto. Buchanans et al. (2000) concluíram não haver comprometimento da eficiência reprodutiva em vacas comprovadamente mais produtoras de leite (Buchanans \& Stutts, 2001) filhas de touros com DEP mais alta para produção de leite.

O sexo dos bezerros não teve efeito sobre a produção de leite das vacas $(\mathrm{P}>0,05)$ nas nove medidas de produção (Tabela 2). Este resultado corrobora os de Melton et al. (1967), Neville et al. (1974), Robinson et al. (1978), Alencar (1987a), Alencar et al. (1988) e Franzo (1997), que também não verificaram efeito do sexo sobre a produção de leite. Independentemente do sexo, o peso dos bezerros é apontado como fonte de variação na produção de leite (Ruttledge et al., 1971; Robinson et al., 1978; Boggs et al., 1980; Alencar et al., 1985; Mallinckrodt et al., 1993), ressaltando-se que a alternância do efeito de sexo em diferentes anos foi estudada por Jeferry et al. (1971). A produção de leite total e a PER também não foram afetadas pelo sexo dos bezerros (Tabela 3).

A raça do touro não influenciou a produção de leite $(\mathrm{P}>0,05)$ nas nove medidas realizadas (Tabela 2$)$, da mesma forma que a PLT e a PER (Tabela 3). Era de se esperar que bezerros cruzas manifestassem o efeito da heterozigose (Koger et al., 1976; Cardellino \& Rovira, 1987), influenciando a produção das vacas por meio de maior consumo de leite (Kress et al., 1990). Entretanto, provavelmente a capacidade de produção de leite das vacas, todas da mesma raça, tenha limitado o maior vigor dos bezerros. Efeito da raça do touro pai sobre a produção de leite (40 e 130 dias) também não foi verificado por Kress et al. (1990), em vacas Hereford, Angus e Simental acasaladas com touros Charolês e Tarentaise.

As médias ajustadas de peso vivo dos bezerros durante a lactação, em períodos de 21 dias, do nascimento à desmama, encontram-se na Tabela 4 . O efeito de ano foi significativo $(\mathrm{P}<0,05)$ em sete das nove medidas realizadas. A ordem de parto, que se confunde com o efeito de ano, apresentou diferença $(\mathrm{P}<0,05)$ apenas aos 84 dias. Os bezerros machos foram mais pesados $(\mathrm{P}<0,05)$ que as fêmeas do nascimento à desmama (exceto 42 e 105 dias), o que também foi verificado por Alencar (1987a), confirmando a influência do sexo no peso do bezerro (Neville, 1962). Franzo (1997), no entanto, 
Tabela 3 - Médias de produção diária de leite $(\mathrm{kg})$ no pico de lactação (42 dias), persistência da lactação (g/dia) e produção de leite total $(P L T)$, ajustadas nos fatores ano, ordem do parto $(\mathrm{VM}=$ multípara, $\mathrm{VP}=$ primípara), prenhez $(\mathrm{P}=$ prenhe, $\mathrm{V}=\mathrm{vazia})$, sexo do bezerro $(\mathrm{M}=$ macho, $\mathrm{F}=$ fêmea $)$ e raça do touro $(\mathrm{N}=$ Nelore, $\mathrm{H}=$ Hereford, $\mathrm{RA}=$ Red Angus)

Table 3 - Mean daily milk yield at lactation peak (42 days) and total milk yield (TMY) adjusted for year, calving order ( $M=$ multiparous, $P=$ primiparous $)$, pregnancy $(P=$ pregnant, $V=$ non pregnant $)$, calf sex $(M=$ male, $F=$ female $)$ and sire breed $(N=$ Nelore, $H=H e r e f o r d$, $R A=\operatorname{Red}$ Angus)

\begin{tabular}{|c|c|c|c|c|}
\hline $\begin{array}{l}\text { Fator } \\
\text { Variable }\end{array}$ & $\mathrm{n}$ & $\begin{array}{l}\text { Pico de lactação ( } \mathrm{kg} / \mathrm{dia}) \\
\text { Lactation peak ( } \mathrm{kg} / \text { day })\end{array}$ & $\begin{array}{ll}\text { Persistência } & (\mathrm{g} / \text { dia }) \\
\text { Persistency } & (\mathrm{g} / \text { day })\end{array}$ & $\begin{array}{l}\text { PLT }(\mathrm{kg}) \\
\text { TMY (kg) }\end{array}$ \\
\hline \multicolumn{5}{|l|}{$\begin{array}{l}\text { Ano } \\
\text { Year }\end{array}$} \\
\hline $\begin{array}{l}1995 \\
1998 \\
2000 \\
2001\end{array}$ & $\begin{array}{l}23 \\
48 \\
42 \\
31\end{array}$ & $\begin{array}{l}9,44 \pm 0,45^{\mathrm{a}} \\
5,98 \pm 0,31^{\mathrm{b}} \\
6,23 \pm 0,34^{\mathrm{b}} \\
6,52 \pm 0,39^{\mathrm{b}}\end{array}$ & $\begin{array}{l}-28,81 \pm 4,61^{\mathrm{a}} \\
-3,36 \pm 3,19^{\mathrm{bc}} \\
-25,84 \pm 3,41^{\mathrm{a}} \\
-14,47 \pm 3,97^{\mathrm{ac}}\end{array}$ & $\begin{array}{c}1186,03 \pm 44,19 \\
1038,31 \pm 30,59 \\
938,67 \pm 32,70 \\
984,56 \pm 38,07\end{array}$ \\
\hline $\begin{array}{l}\mathrm{VM}(M) \\
\mathrm{VP}(P)\end{array}$ & $\begin{array}{l}69 \\
75\end{array}$ & $\begin{array}{l}8,27 \pm 0,26^{\mathrm{a}} \\
5,81 \pm 0,25^{\mathrm{b}}\end{array}$ & $\begin{array}{l}-24,01 \pm 2,66 \\
-12,23 \pm 2,55\end{array}$ & $\begin{array}{l}1158,34 \pm 25,52^{\mathrm{a}} \\
915,44 \pm 24,47^{\mathrm{b}}\end{array}$ \\
\hline \multicolumn{5}{|c|}{$\begin{array}{l}\text { Prenhez } \\
\text { Pregnancy }\end{array}$} \\
\hline $\begin{array}{l}\mathrm{F} \\
\mathrm{M}\end{array}$ & $\begin{array}{l}88 \\
56\end{array}$ & $\begin{array}{l}6,85 \pm 0,23 \\
7,24 \pm 0,29\end{array}$ & $\begin{array}{l}-18,20 \pm 2,36 \\
-18,04 \pm 2,95\end{array}$ & $\begin{array}{l}1018,26 \pm 22,59 \\
1055,52 \pm 28,32\end{array}$ \\
\hline \multicolumn{5}{|c|}{$\begin{array}{l}\text { Raça touro } \\
\text { Sire breed }\end{array}$} \\
\hline $\begin{array}{l}\mathrm{H} \\
\mathrm{N} \\
\mathrm{RA} \\
\text { Geral } \\
\text { Overall }\end{array}$ & $\begin{array}{c}46 \\
82 \\
16 \\
144\end{array}$ & $\begin{array}{c}6,17 \pm 0,32 \\
7,31 \pm 0,24 \\
7,64 \pm 0,54 \\
7,04\end{array}$ & $\begin{array}{c}-21,53 \pm 2,44 \\
-23,73 \pm 3,26 \\
-9,10 \pm 5,52 \\
-18,12\end{array}$ & $\begin{array}{c}988,60 \pm 31,25 \\
1025,85 \pm 23,41 \\
1096,22 \pm 52,99 \\
1036,89\end{array}$ \\
\hline
\end{tabular}

Letras diferentes na coluna, dentro de fatores, indicam médias diferentes $(P<0,05)$.

Means followed by different letters in the same column differ $(P<0.05)$.

não verificou efeito do sexo no peso dos bezerros dentro de grupos genéticos. A gestação teve efeito significativo $(\mathrm{P}<0,05)$ aos 105 e 147 dias de lactação e no peso corrigido à desmama, mesmo sem diferença na produção de leite nesse período entre gestantes e não-gestantes.

O sistema para expressar as exigências de energia líquida (EL) para crescimento e terminação em bovinos de corte foi desenvolvido por Lofgreen \& Garrett (1968) e sua utilização associada a escores de condição corporal e à produção de leite (Bartle et al. 1984, Buskirk et al., 1992), é atualmente a forma mais indicada para se avaliar o aporte energético para os animais. O valor energético do leite muitas vezes é subestimado em programas que visam sua substituição por outro alimento, retirando do bezerro em idades muito precoces uma fonte energética e protéica de altíssimo valor biológico. Os resultados de produção de leite e desempenho dos bezerros neste estudo permitiram calcular, com base no estudo de Robison et al. (1978), a utilização e a disponibilidade de EL do leite para os bezerros. A produção média diária de leite durante a lactação foi de $5,34 \mathrm{~kg}$ e o ganho de peso médio diário, de 0,669 kg, sendo necessários, em média, $8,12 \mathrm{~kg}$ de leite para cada $\mathrm{kg}$ de ganho de peso.

A relação entre a produção de leite e o ganho de peso dos bezerros, bem como a avaliação da energia líquida para ganho disponível no leite durante a lactação com base nas médias ajustadas, encontram-se na Tabela 5 .

A eficiência na utilização do leite pelo bezerro é mais baixa no pico e aumenta à medida que transcorre a lactação, possivelmente em razão da complementação da dieta com forragem (Boggs et al., 1980). Porém, o resultado mais importante apresentado na Tabela 5 é a disponibilidade de energia líquida para ganho no leite, que se manteve positiva até o quarto mês de lactação, atendendo à mantença com sobra para ganho. Apenas a partir do quinto mês os valores de ELg começaram a ser negativos, evidenciando a importância do leite na alimentação do bezerro e comprovando a dificuldade na substituição por forragem, sem considerar o valor biológico da proteína a ser utilizada. Estes resultados 


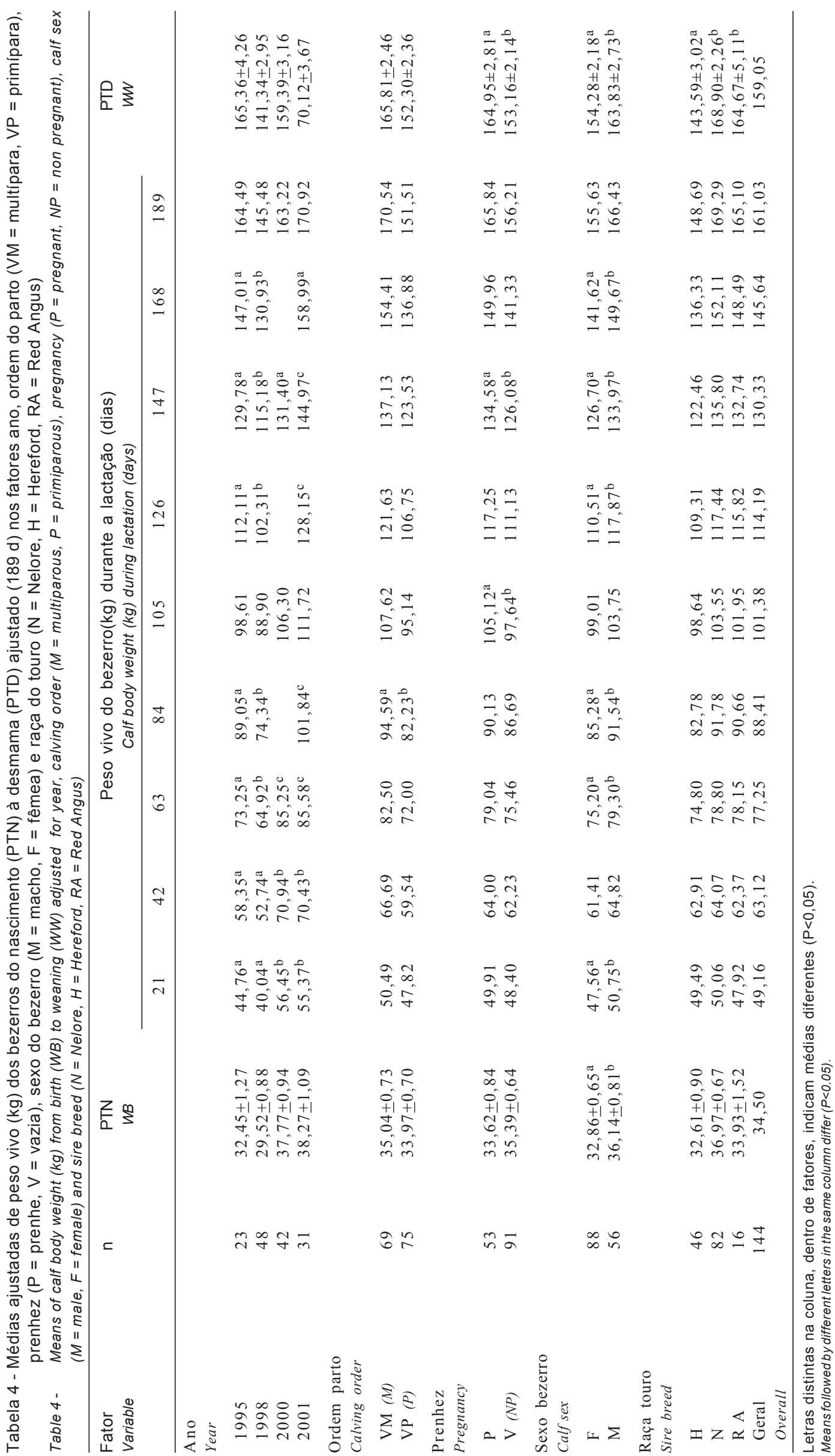


Tabela 5 - Produção de leite e ganho médio diário (GMD) de bezerros, exigências de energia líquida de mantença (ELm) e ganho (ELg) e energia líquida disponível para ganho no leite

Table 5 - $\quad$ Milk yield and average daily gain (ADG) of calves, net energy requirements for maintenance (NEm) and gain (NEg) and milk net energy available for gain

\begin{tabular}{|c|c|c|c|c|c|c|c|c|}
\hline \multirow[t]{2}{*}{$\begin{array}{l}\text { Dias de lactação } \\
\text { Lactation days }\end{array}$} & \multirow[t]{2}{*}{$\begin{array}{l}\text { Peso vivo }(\mathrm{kg}) \\
\text { Body weight }(\mathrm{kg})\end{array}$} & \multirow{2}{*}{$\begin{array}{c}\text { Produção de } \\
\text { leite }(\mathrm{kg}) \\
\text { Milk yield }(\mathrm{kg})\end{array}$} & \multirow{2}{*}{$\begin{array}{c}\text { GMD } \\
\text { bezerro }(\mathrm{kg}) \\
\text { Calves } A D G(\mathrm{~kg})\end{array}$} & \multirow[t]{2}{*}{$\begin{array}{l}\text { Leite/ganho (kg) } \\
\text { Milk/gain (kg) }\end{array}$} & \multicolumn{2}{|c|}{$\begin{array}{l}\text { Exigências de EL (Mcal) } \\
\text { NE requirements (Mcal) }\end{array}$} & \multicolumn{2}{|c|}{$\begin{array}{l}\text { ELg disponível no leite } \\
\text { Milk available } N E g\end{array}$} \\
\hline & & & & & $\begin{array}{l}\text { Elm } \\
N E m\end{array}$ & $\begin{array}{l}\text { Elg } \\
\quad N E g\end{array}$ & $\%$ & Mcal \\
\hline 21 & 49,15 & 5,62 & 0,698 & 8,06 & 1,43 & 0,74 & 0,72 & 97,30 \\
\hline 42 & 63,11 & 7,04 & 0,665 & 10,59 & 1,72 & 0,85 & 0,93 & 109,41 \\
\hline 63 & 77,25 & 5,91 & 0,673 & 8,78 & 2,01 & 1,01 & 0,54 & 53,47 \\
\hline 84 & 88,41 & 5,68 & 0,531 & 10,69 & 2,22 & 0,86 & 0,39 & 45,35 \\
\hline 105 & 101,38 & 5,3 & 0,618 & 8,58 & 2,46 & 1,12 & 0,20 & 17,86 \\
\hline 126 & 114,19 & 5,11 & 0,610 & 8,38 & 2,69 & 1,21 & 0,05 & 4,13 \\
\hline 147 & 130,33 & 4,78 & 0,769 & 6,22 & 2,97 & 1,72 & $-0,15$ & $-8,72$ \\
\hline 168 & 145,64 & 4,25 & 0,729 & 5,83 & 3,23 & 1,76 & $-0,38$ & $-21,59$ \\
\hline 189 & 161,02 & 4,39 & 0,732 & 5,99 & 3,48 & 1,91 & $-0,46$ & $-24,08$ \\
\hline
\end{tabular}

Exigências de energia líquida para mantença e ganho pelos bezerros, com base em seus respectivos pesos, ganhos e consumo de leite, calculadas utlizando-se fórmulas e valores retirados do NRC $(1970,1996)$.

Net energy for maintenance and gain required by calves, based on their weights, gains and milk intake were calculated from NRC (1970,1996) tables.

são semelhantes aos verificados por Robison et al. (1978), ao estudarem a lactação de vacas Hereford utilizando metodologia de cálculos semelhante.

\section{Conclusões}

A produção quantitativa de leite em vacas de corte sob condições extensivas tem amplitude limitada e não sofre interferência de efeitos como sexo, raça do bezerro ou gestação. Entretanto, é afetada pela ordem do parto (a maior diferença ocorre no início da lactação), de modo que vacas adultas apresentam maiores picos de lactação. $\mathrm{O}$ leite atende plenamente às exigências energéticas no início da lactação. As exigências de mantença, por sua vez, são atendidas até o quarto mês, com pequena sobra para ganho de peso.

\section{Literatura Citada}

ALENCAR, M.M. Efeitos da produção de leite sobre o desenvolvimento de bezerros Canchim. Revista da Sociedade Brasileira de Zootecnia, v.16, n.1, p.01-13, 1987a.

ALENCAR, M.M. Herdabilidade e efeito da produção de leite sobre a eficiência reprodutiva de vacas da raça Canchim. Revista da Sociedade Brasileira de Zootecnia, v.16, n.2, p.163-169, $1987 b$.

ALENCAR, M.M.; CRUZ, G.M.; TULLIO, R.R. et al. Comparação de diferentes equações para caracterizar a curva de lactação em bovinos de corte. Revista da Sociedade Brasileira de Zootecnia, v.24, n.4, p.530-541, 1995.

ALENCAR, M.M.; JUNQUEIRA FILHO, A.A.J.; PARANHOS, N.E. Produção de leite em vacas da raça Canchim. Revista da Sociedade Brasileira de Zootecnia, v.14, n.3, p.358-366, 1985.

ALENCAR, M.M.; OLIVEIRA, F.T.T; TAMBASCO, A.J. et al. Desenvolvimento pós-desmama e eficiência reprodutiva pósparto em gado de corte: Influência da produção de leite. Revista da Sociedade Brasileira de Zootecnia, v.22, n.6, p.1012$1018,1993$.
ALENCAR, M.M.; RUZZA, F.J.; PORT, E.J.S. Desempenho produtivo de fêmeas das raças Canchim e Nelore. III. Produção de leite. Revista da Sociedade Brasileira de Zootecnia, v.17, n.4, p.317-328, 1988.

ALENCAR, M.M.; TULLIO, R.R.; CRUZ, G.M. et al. Produção de leite da vaca e desenvolvimento do bezerro em gado de corte. Revista da Sociedade Brasileira de Zootecnia, v.25, n.1, p.92-101, 1996.

BARTLE, S.J.; MALES, J.R.; PRESTON, R.L. Effect of energy intake on the postpartum interval in beef cows and the adequacy of the cow's milk production for calf growth. Journal of Animal Science, v.58, n.5, p.1068-1074, 1984.

BAUMAN, D.E. Regulation of nutrient partioning during lactation: homeostasis and homeoresis. In: CRONJÉ, P.B. (Ed.) Ruminant physiology. Digestion, metabolism, growth and reproduction. Wallingford: CAB International, 2000. p.311-328.

BEAL, W.E.; NOTTER, D.R.; AKERS, R.M. Techniques for estimation of milk yield in beef cows and relationships of milk yield to calf weight gain and postpartum reproduction. Journal of Animal Science, v.68, p.937-943, 1990.

BOGGS, D.L.; SMITH E.F.; SCHALLES, R.R. et al. Effects of milk and forage intake on calf performance. Journal of Animal Science, v.51, n.3, p.550-553, 1980.

BUCHANAN, D.S.; STUTTS, K.J.; WETTEMANN, R.P. Genetic variation in days to onset of post partum ovarian function for cows sired by high and low milk EPD Hereford and Angus bulls. http://www.ansi.okstate.edu/ research/2000rr/01.htm

BUCHANAN, D.S.; STUTTS, K.J. Evaluation of milk production and calf performance in range beef cows sired by high and low milk expected progeny difference Angus and Hereford bulls. http://www.ansi.okstate.edu/research/2001 rr/01/01.htm

BUSKIRK, D.D.; LEMENAGER, R.P.; HORSTMAN, L.A. Estimation of netenergy requeriments $\left(\mathrm{NE}_{\mathrm{m}}\right.$ and $\left.\mathrm{NE}_{\mathrm{D}}\right)$ of lacting beef cows. Journal of Animal Science, v.70, p.3867-3876, 1992.

BUTLER, W.R. Nutritional interactions with reproductive performance in dairy cattle. Animal Reproduction Science, v.60-61, p.449-457, 2000.

CARDELLINO, R.A.; ROVIRA, J. Mejoramiento genético animal. Montevideo: Agropecuaria Hemisferio Sur, 1987. 253p.

COLE, L.J.; JOHANSSON, I. The yield and composition of milk from Aberdeen Angus cows. Journal of Dairy Science, v.6, p.565-580, 1933 .

COOK, A.C.; BLACK, W.H.; BRADFORD JR., K. et al. Relationship of milk production of milking Shorthorn dams to the carcass 
quality of their steer progeny. Journal of Animal Science, v.1, n.4, p.345, 1942 (abstract).

DAWSON, W.M.; COOK, A.C.; BRADFORD, K.Jr. Milk production of beef Shorthorn cows. Journal of Animal Science, v.19, n.1, p.502-508, 1960.

DAY, M.L.; IMAKAWA, K.; CLUTTER, A.C. et al. Suckling behavior of calves with dams varying in milk production. Journal of Animal Science, v.65, p.1207-1212, 1987.

DILLARD, E.V.; YUSUFF, M.K.M.; ROBISON, O.W. Milk production in Hereford cows. II. Heritabilities and repeatabilities. Journal of Animal Science, v.47, n.1, p.137-141, 1978

DREWRY, K.J.; BROWN, C.J.; HONEA, R.S. Relationships among factors associated with mothering ability in beef cattle. Journal of Animal Science, v.18, n.3, p.938-946, 1959.

FRANZO, V. Produção de leite em vacas primíparas de corte e sua relação com o desenvolvimento dos terneiros. Pelotas: Universidade Federal de Pelotas, 1997. 102p. Dissertação (Mestrado em Zootecnia) - Universidade Federal de Pelotas, 1997.

FRANZO, V.; PIMENTEL, M.A.; JARDIM, P.O. et al. Produção de leite em vacas de corte e desenvolvimento ponderal de seus terneiros. In: REUNIÃO ANUAL DA SOCIEDADE BRASILEIRA DE ZOOTECNIA, 34., 1997, Juiz de Fora. Anais... Juiz de Fora: Sociedade Brasileira de Zootecnia, 1997. p.242-244.

GREEN, R.D.; CUNDIFF, L.V.; DICKERSON, G.E. et al. Output/ input differences among nonpregnant, lactating Bos indicus Bos taurus and Bos taurus - Bos taurus F1 cross cows. Journal of Animal Science, v.69, p.3156-3166, 1991

JEFFERY, H.B.; BERG, R.T.; HARDIN, R.T. Factors influencing milk yield of beef cattle. Canadian Journal of Animal Science, v.51, n.3, p.551-560, 1971.

JENKINS, T.G.; FERRELL, C.L. Lactation characteristics of nine breeds of cattle fed various quantities of dietary energy. Journal of Animal Science, v.70, p.1652-1660, 1992.

JENKINS, T.G.; FERREL, C.L.; ROBERTS A.J. Lactation and calf weight traits of mature crossbred cows fed varying daily levels of metabolizable energy. Journal of Animal Science, v.78, p. 7-14, 2000 .

JOHNSON, C.R.; LALMAN, D.L.; BROWN, M.A. et al. Influence of milk production potential on forage dry matter intake by multiparous and primiparous Brangus females. Journal of Animal Science, v. 81, p.1837-1846, 2003.

KOGER, M.; CUNHA, T.J.; WARNICK, A.C. Cruzamientos en ganado vacuno de carne. Montevideo: Agropecuaria Hemisferio Sur, 1976. 559p.

KRESS, D.D.; DOORNBOS, D.E.; ANDERSON, D.C. Performance of crosses among Hereford, Angus and Simmental cattle with different levels of Simmental breeding: V. Calf production, milk production and reproduction of three - to eight - year - old dams. Journal of Animal Science, v.68, p.1910-1921, 1990.

LALMAN, D.L.; WILLIAMS, J.E.; HESS, B.W. et al. Effect of dietary energy on milk production and metabolic hormones in thin, primiparous beef heifers. Journal of Animal Science, v.78, p.530-538, 2000 .

LEAL, T.C.; FREITAS, J.E. Correlação entre produção de leite e ganho de peso de terneiros da raça Charolesa. Anuário Técnico IPZFO, v.9, p.91-101, 1982.

LOFGREEN, G.P.; GARRETT, W.N. A system for expressing net energy requirements and feed values for growing and finishing beef cattle. Journal of Animal Science, v.27, n.3 p.793-806, 1968.

MACEDO, W. Levantamento de reconhecimento dos solos do município de Bagé, RS. Bagé: Embrapa Pecuária Sul, 1984. 69p.

MALLINCKRODT, C.H.; BOURDON, R.M.; GOLDEN, B.L. et al. Relationship of maternal milk expected progeny differences to actual milk yield and calf weaning weight. Journal of Animal Science, v.71, p.355-362, 1993.

McMORRIS, M.R.; WILTON, J.W. Breeding system, cow weight and milk yield effects on various biological variables in beef production. Journal of Animal Science, v.63, p.13611372,1986
MELTON, A.A.; RIGGS, J.K.; NELSON, L.A. et al. Milk production, composition and calf gains of Angus, Charolais and Hereford cows. Journal of Animal Science, v.26, n.4, p.804-809, 1967.

MENDONÇA G.; PIMENTEL, M.A.; OSÓRIO, J.C. et al. Produção de leite em bovinos de corte 4. Relação entre produção de leite de vacas Hereford, peso ao parto e desenvolvimento dos terneiros. In: REUNIÃO ANUAL DA SOCIEDADE BRASILERA DE ZOOTECNIA, 38., 2001, Piracicaba. Anais... Piracicaba: Sociedade Brasileira de Zootecnia, 2001. p. 453-454

MILLER, S.P.; WILTON, J.W.; PFEIFFER, W.C. Effects of milk yield on biological efficiency and profit of beef production from birth to slaughter. Journal of Animal Science, v.77, p.349-352, 1999.

NEVILLE JR., W.E. Influence of dam's milk production and other factors on 120 and 240-day weight of Hereford calves. Journal of Animal Science, v.21, n.2, p.315-320, 1962.

NEVILLE JR., W.E.; WARREN, E.P.; GRIFFEY, W.A. Estimates of age effects on milk production in Hereford cows. Journal of Animal Science, v.38, n.1, p.1-10, 1974

NCSS 6.0. Statistical System for Windows-User's Guide I, II, II Kaysville, Utah, 1996. 2204p.

NATIONAL RESEARCH COUNCIL - NRC. Nutrient requirements of beef cattle. Washington, D.C. National Academy Press, 1970. 77p.

NATIONAL RESEARCH COUNCIL - NRC. Nutrient requirements of beef cattle. 7.ed. Washington, D.C. National Academy Press, 1996. 241p.

PIMENTEL, M.A.; FRANZO, V.; MENDONÇA, G. et al. Produção de leite em bovinos de corte III. Peso ao parto e eficiência individual em novilhas Hereford e cruzas. In: REUNIÃO ANUAL DA SOCIEDADE BRASILERA DE ZOOTECNIA, 38., 2001, Piracicaba. Anais... Piracicaba: Sociedade Brasileira de Zootecnia, 2001. p.393-394

PIMENTEL, M.A.; MORAES, J.C.; JAUME, C.M. et al. Produção de leite em bovinos de corte. 5. Fertilidade pós-parto em vacas Hereford In: REUNIÃO ANUAL DA SOCIEDADE BRASILERA DE ZOOTECNIA, 39., 2002, Recife. Anais... Recife: Sociedade Brasileira de Zootecnia, 2002. CD-ROM

ROBISON, O.W.; YUSUFF, M.K.M. Milk production in Hereford cows I. Means and correlations. Journal of Animal Science, v.47, n.1, p.131-136, 1978.

RUTLEDGE, J.J.; ROBISON, O.W.; AHLSCHWEDE, W.T. et al. Milk yield and its influence on 205 - day weigth of beef calves. Journal of Animal Science, v.33, n.3, p.563-567, 1971.

SOUZA, C.J.H.; MORAES, J.C.F. Manual de sincronização de cio em ovinos e bovinos. Bagé: Embrapa Pecuária Sul, 1998. 76p.

SILVEIRA, C.L.M.; SALOMONI, E.; MARTINS, C.A.; et al. Avaliação da produção de leite em vacas Hereford paridas em distintas estações do ano. Revista Científica Rural, v.4, n.1, p.109-118, 1999.

SHORT, R.E.; BELLOWS, R.A.; STAIGMILLER, J.G. et al. Physiological mechanisms controlling anestrus and infertility in pospartum beef cattle. Journal of Animal Science, v.68, n.3, p.799-815, 1990

TOTUSEK, R.; DUDLEY A. Estimators of milk production in beef cows. Journal of Animal Science, v.24, n.3, p.906, 1965 (abstract).

WILLIAMS, J.H.; ANDERSON, D.C.; KRESS, D.D. Milk production in Hereford cattle. I. Effects of separation interval on weight suckle - weight milk production estimates. Journal of Animal Science, v.49, n.6, p.1438-1442, 1979a.

WILLIANS, J.H.; ANDERSON, D.C.; KRESS, D.D. Milk production in Hereford cattle. II. Physical measurements: repeatabilities and relationships with milk production. Journal of Animal Science, v.49, n.6, p.1443-1448, 1979b

WISTRAND, G.C.; RIGGS, J.K. Milk production of Santa Gertrudis cows as measured by calf nursing and machine milking methods. Journal of Animal Science, v.25, n.1, p.263-264, 1966 (abstract). 